\title{
Biomedical Applications of the Discrete Wavelet Transform
}

\author{
Raquel Cervigón \\ Universidad de Castilla-La Mancha \\ Spain
}

\section{Introduction}

In eighties wavelets came up as the time-frequency revolution in signal processing. In 1989 Mallat proposed the fast Discrete Wavelet Transform (DWT) algorithm to decompose a signal using a set of quadrature mirror decomposition filters, and which have respective band-pass and low-pass properties specific to each mother wavelet (Mallat, 1999). Since this period Wavelets have been applied in a variety of fields including fluid dynamics, engineering, finance geophysics, study of musical tones, image compressionand de-noising just to name few. In addition, it has been extensively used in medicine because of the irregularities inherent to biological signals.

In the discrete wavelet analysis the information stored in the wavelets coefficient is not repeated, it allows the complete regeneration of the original signal without redundancy. This property has motivated much of the effort for development of wavelet-based signal compression algorithms, particularly for ECG signals compression techniques are important to enlarge storage capacity an improve methods of ECG data transmission. DWT removes redundancy in the signal and provides a high compression ratio and high quality reconstruction of ECG signal.

The bioelectric signals contain noise originated by devices or interference of the network that hardly can be eliminated by conventional analogous filters. DWT is a technique to filtrate signals with low distortion to eliminate noise. This process can be applied to different physiology signals, where signals with additive noise are decomposed using the DWT and a threshold is applied to each of the detail coefficient levels. All coefficients with an absolute value greater than the threshold are thought to be part of information and those below the threshold are presumably derived from noise. The noise coefficients can be set to zero and a noise-free signal can then be reconstructed and used for signal detection. Recently, several wavelet-based methods have been used for unsupervised de-noising and detection of data with low signal-to-noise ratio. In particular, DWT has been applied in the quantification of human sympathetic nerve signal activity to discriminate action potentials. Wavelet decomposition effectively filters the nerve signal into several frequency sub-bands while preserving its temporal structure. Each sub-band of wavelet processing decorrelates successive noise-related values and compares progressively more dilated versions of a general spike shape to each point in the signal. This process can make easier the detection of action potentials by separating the signal and noise using their distinct time-frequency signatures. 
Discrete Wavelet analysis corresponds to windowing in a new coordinate system, in which space and frequency are simultaneously localized; this property can be helpful in pattern extraction. Wavelets as an alternative tool to analyze non-stationary signal have been applied to ECG delination, to detect accurately the different waves forming the entire cardiac cycle, especially in areas of limited perfomance of of current techniques like QT and ST intervals, P and T-wave recognition, and to clasify ECG waves in different cardiopatologies, identifying ECG waveforms from different arrhythmias, or discriminating between normal and anormal cardiac pattern. In addition, DWT is able to detect specific detailed time-frequency components of ECG signal, for instance, the registers which are sensitive to transient ischemia and eventual restoration of electrohysiological funtion of the myocardial tissue. Moreover, methods for analysing heart rate variability using wavelet transform can be used to detect transient changes without losing frequency information. Several authors have successfully demonstrated the utility of the DWT in time-varying spectral analysis of heart-rate variability during dynamic cardiovascular stimulation.

\section{Discrete Wavelet Transform}

DWT is a fast algorithm for machine computation, like the Fast Fourier Transform (FFT), it is linear operation that operates on a data vector, transforming it into a numerically different vector of the same length. Also like the FFT, the DWT is invertible and orthonormal. In the FFT, the basis functions are sines and cosines. In the DWT, they are hierarchical set of "wavelet functions" that satisfy certain mathematical criteria (Daubechies, 1992; Mallat, 1989b) and are all translations and scalings of each other.

There is an even faster family of algorithms based on a completely different idea, namely that of multiresolution analysis, or MRA (Mallat, 1989a), then the whole construction may be transcripted into a pair of quadrature mirror filters, defined from the underlying wavelet function, and both are applied to the signal and down-sampled by a factor of two. This process splits the signal into two component, each of half the original length, with one containing the low-frequency or "smooth" information and the other the high-frequency or "difference" information. The process is performed again on the smooth component, breaking it up into "low-low" and "high-low" components and it is repeated several times.

DWT achieves a multiresolution decomposition of $x_{n}$ on $J$ octaves labelled by $j=1, \ldots, J$. It is precisely this requirement for a multresolution-hence hierarchical- structure that makes fast computation possible. The requirement for a multiresolution computation can be stated as follows: Given some signal, at scale $j$, one decomposes it in a sum or details, at scale $j+1$ (the true wavelet coefficients), plus some residual, representing the signal at resolution $j+1$ (twice as coarse). A further analysis at coarser scales involves only the residual.

Consider two filter impulse responses $g(n)$ (corresponding to some low-pass interpolating filter-the scaling function) and $h(n)$ (corresponding to some a high-pass filter-the discrete wavelet) (eq. 1 and 2). The downsampled outputs of first low-pass and high-pass filters provide the approximation, and the detail, respectively. The first approximation is further decomposed and this process is continued until all wavelet coefficients are determined.

$$
\begin{aligned}
& h_{j+1}(n)=\sum_{k} h_{j}(k) g[n-2 k] \\
& g_{j+1}(n)=\sum_{k} g_{j}(k) h[n-2 k]
\end{aligned}
$$


The wavelets and scaling sequences are obtained iteratively as i.e., one goes from one octave $j$ to the next $(j+1)$ by applying the interpolation operator

$$
f(n) \rightarrow \sum_{k} f(n) g(n-2 k)
$$

Which should be thought of as the discrete equivalent to the dilation $f(t) \rightarrow 2^{-1 / 2} f(t / 2)$.

Consider, for example, the computation of wavelet coefficients $c_{j, k}$, for a fixed $j$, the coefficient is the result of filtering the input signal by $h_{j}(n)$ and decimating the output by the suppression of one every $2^{j}$ th sample. Now the z-transform of filter $h_{j}(n)$ can be easily deduced from equation 1 , which reads $H_{j+1}(z)=H_{j}\left(z^{2}\right) G(z)$ in z-transform notation. We obtain:

$$
H_{j+1}(z)=G(z) G\left(z^{2}\right) \ldots G\left(z^{2^{j-1}}\right) H\left(z^{2 j}\right)
$$

and similarly for $g_{j}(n)$,

$$
G_{j+1}(z)=G(z) G\left(z^{2}\right) \ldots G\left(z^{2^{j}}\right)
$$

The computations of a DWT are easily reorganized in form of binary tree, where the decomposition may also be truncated at any level of the process before an average signal of length of one sample is reached. In any event, the dyadic DWT consists of the set of detail signals generated at each level of the transform, together with the average signal generated at the highest level (shortest length signals) of the transform.

A remarkable feature of many useful wavelet transforms, is that they obey a perfect reconstruction theorem. That is the dyadic DWT may be inverted to recover the original signal exactly. The inversion process is carried out first by upsampling (or expanding) the highest level detail and average signals. Upsampling is carried out by inserting zeros between samples of the signal to be upsampled. Then, the upsampled average and detail signals are run through synthesis filters and added together. The sum signal is the average signal for the next lowest level of the wavelet transform. This process is carried out at each lower level until the original signal is recovered at the lowest level as the zero level average signal (Kaiser, 1994; Mallat, 1998; Strang \& Nguyen, 1997).

The computed wavelet coefficients provide a compact representation that shows the energy distribution of the signal in time and frequency. We assume that the signals are stationary within each short segment in time. Thus within the segment, the variance of the wavelet transform $w x(t)$ and the wavelet function $\psi(t)$ can be considered as a value unrated to $t$, written as,

$$
E[x \psi(t)]^{2}=E\left[(x * \psi)^{2}(t)\right]=\sigma_{x}^{2}
$$

And in the frequency domain,

$$
\sigma_{x}^{2}=\frac{1}{2 \pi} \int_{-\infty}^{\infty} S_{x}(\omega)|\psi(\omega)|^{2} d \omega
$$

Furthermore, the spectral components of interest may be located anywhere in the frequency axis, even in the neighborhood of the cross-point between two adjacent frequency bands. At this location, the spectral component is assigned with a small gain signalling, and low detection sensitivity. This problem can be approached by considering the cross-correlation between $w x_{j}(t)$ and $w x_{j+1}(t), R w x_{j}, w x_{j+1}=E\left[w x_{j}(t+1) w x_{j+1}(t)\right]$ and the autocorrelation of the signal. 


\section{Discrete Wavelet Transform in biomedical research}

Wavelet Transform has been proposed as an alternative way to analyze the non-stationary biomedical signals, which expands the signal onto the basis functions. The wavelet method act as a mathematical microscope in which we can observe different parts of the signal by just adjusting the focus.

A conventional application of wavelet methods to processing of a medical waveform uses a wavelet transform based on the application of a single wavelet, rather than a basis set constructed from a family of mathematically related wavelets. Again, the choice of a wavelet with appropriate morphological characteristics relative to the physiological signal under consideration is crucial to the success of the application. In the following sections will be introduced different uses of DWT in cardiology research, with interesting applications such as de-noising and compression of medical signals, electrocardiogram (ECG) segmentation and feature extraction, analysis of heart rhythm variability, and the analysis of different cardiac arrhythmias.

\section{Signal compression}

The compressibility of a sampled signal is the radio of the total area of time-frequency plane $(N$, for a signal sampled at $N$ ) divides by the total area of the information cells. It is possible to automatically analyze signals by expanding them in the best basis, then drawing the corresponding time-frequency plane representation.

The DWT is both "complete" and has "zero redundancy", which means that all the signal information is contained in the resulting transform and none is duplicated between transform coefficients. By converting the signal into its DWT coefficients and then removing all except those containing the most pertinent signal information, the resulting transform is much smaller in size, which provides a good way of compressing a signal. Performing an "inverse transform" on the remaining components recreates a signal that very nearly matches the original. This is the basis of compression algorithms that can be applied to biomedical images and signals, such as in the development of effective ECG data compression. Increasing use of computerized ECG processing systems requires effective ECG data compression techniques which aim to enlarge storage capacity and improve methods of ECG data transmission over internet lines. Moreover ECG signals are collected both over long periods of time and at high resolution. This creates substantial volumes of data for storage and transmission. The fundamental reason that ECG compression is regarded as a difficult problem is that the ECG waveform contains clinically significant information on a wide variety of time scales. Data compression seeks to reduce the number of bits of information required to store or transmit digitized ECG signals without significant loss of signal quality. Moreover, some ECG compression algorithms have been used only for strictly limited diagnostic objectives, as in Holter monitors. Another objective is to develop a high-fidelity compression algorithm that would not impair later physician diagnoses.

An early paper suggested the wavelet transform as a method for compressing both ECG and heart rate variability data sets (Crowe et al., 1992). Thakor et al. compared two methods of data reduction on a dyadic scale for normal and abnormal cardiac rhythms, detailing the errors associated with increasing data reduction ratios (Thakor et al., 1993). Using DWT and Daubechies D10 wavelets, Chen et al. compressed ECG data sets resulting in compression ratios up to 22.9:1 while retaining clinically acceptable signal quality, with an adaptive quantization strategy which allows a predetermined desired signal quality to be 
achieved (Chen \& Itoh, 1998). Miaou et al. (Miaou \& Larn, 2000) also propose a quality driven compression methodology based on Daubechies wavelets and later on biorthogonal wavelets (Miaou \& Lin, 2002), this algorithm adopts the set partitioning of hierarchical tree (SPIHT) coding strategy.

\section{Wavelet Transform based filtering "De-noising"}

The noise present in the signal can be removed by applying the wavelet shrinkage de-noising method while preserving the signal characteristics, regardless of its frequency content.

Wavelets have the added advantage that the resulting expansions are orthogonal or energy preserving, allowing to compare an adapted expansion to signals in order to minimize the cost of representation. Such adapted decompositions perform compression and analysis simultaneously. It is possible to design an idealized graphical presentation of the time-frequency information obtained by such a best adapted wavelet analysis, and for such presentation is possible to recognize and extract transient features. The small components in the analysis may be treated as noise and discarded, where an iterative algorithm always produces the best decomposition, at the cost of many more iterations plus more work for each iteration. Mallat's stopping criterion is to test the amplitude ratio of successive extracted amplitudes; this is a method of recognizing residuums which have the statistics of random noise.

Consider the standard univariate regression: $y_{i}=f\left(x_{i}\right)+\epsilon_{i}$, where $i=1, \ldots, n$, and $\epsilon_{i}$ are independent $N\left(0, \sigma^{2}\right)$ random variables; and $f$ is the "true" function. We can reformulate the problem in terms of wavelet coefficients: $\hat{w}_{j k}=w_{j k}+\epsilon_{j k}$, where $j$ is the level $(j=$ $0, \ldots, j-1)$, and $k$, the displacement $\left(k=0, \ldots, 2^{j}\right)$. It is often reasonable to assume that only a few large coefficients contain information about the underlying function, while small coefficients can be attributed to noise. Shrinkage consists in attenuating or eliminating the smaller wavelet coefficients and reconstructing the profile using mainly the most significant wavelet coefficients and all the scaling coefficients. Several shrinkage approaches have been proposed. For example, the "hard" threshold approach selects coefficients using a keep or kill policy, nevertheless using "soft" thresholding, if the magnitude of the wavelet coefficient is greater than (less than, respectively) the threshold, the coefficient is shrunk toward zero by an amount that depends on how large the magnitude of the coefficient is (set to zero, respectively). Donoho and Johnstone proposed the "universal" threshold, $\lambda=\sigma \sqrt{2 \log n}$, and showed that it performs very well in both hard and soft thresholding. Thresholds can also be chosen based on the data using a hypothesis testing procedure (Alshamali \& Al-Fahoum, 2003; Donoho \& Johnstone, 1994). Data-adaptive thresholds might become very important in analyzing molecular biological data because hypothesis testing procedures can be used to test the appropriateness of various thresholds to the data under different biological assumptions (Lio, 2003). Finally, it is worth mentioning that several authors have proposed Bayesian thresholds and have reported interesting results (Abramovich et al., 2009).

This evolution in electrocardiographic start with the algorithms for noise reduction in ECG signals using the dyadic wavelet transform with wavelet-based and wavelet packet-based thresholding methods for removing noise from the ECG (Kishimoto et al., 1995; Tikkanen, 1999).

More recently, Nikolaev et al have suppressed electromyogram (EMG) noise in the ECG using a method incorporating wavelet transform domain Wiener filtering (Nikolaev et al., 2001), this method resulted in an improvement in signal-to-noise ratio of more than $10 \mathrm{~dB}$. 
In addition, the non-invasive blood pressure artifact removal algorithm makes use of DWT. The system used in most patient monitors measures the small fluctuations in pressure in a blood pressure cuff (applied to one of the patient's limbs) to obtain a determination of the patient's systolic and diastolic pressure. Usually the mean arterial pressure and pulse rate are obtained as well. These pressure fluctuations are usually termed "oscillometric pulses" (Geddes \& Badylak, 1991). The wavelet-based artifact elimination algorithm is based on the observation that the dyadic DWT puts the physiologic oscillometric waveform in a very different region of the transform plane than the signal components attributable to artifact. The modified DWT may then be inverted to yield a reconstruction of the oscillometric signal with artifact substantially reduced. The reconstructed oscillometric signal may then be used as an input to a pressure determination algorithm in the usual way for the measurement of desired patient pressure values.

\section{ECG signal parameter extraction}

The ECG registers a measure of the electrical activity associated with the heart. The ECG is measured at the body surface and results from electrical changes associated with activation first of the two small heart chambers, the atria, and then of the two larger heart chambers, the ventricles. The contraction of the atria manifests itself as the $P$ wave in the ECG and contraction of the ventricles produces the feature known as the $Q R S$ complex. The subsequent return of the ventricular mass to a rest state repolarization produces the $T$ wave. Repolarization of the atria is, however, hidden within the dominant QRS complex. Analysis of the local morphology of the ECG signal and its time varying properties has produced a variety of clinical diagnostic tools.

To use ECG signals as identity verification, a real-time detection of the ECG characteristics is needed. With the real-time extraction of ECG characteristics, we could verify different individual. The basic objects of the analysis are a P-wave, a QRS-complex, a T-wave, a P-Q interval, a S-T segment, and a Q-T interval (see Fig. 1).

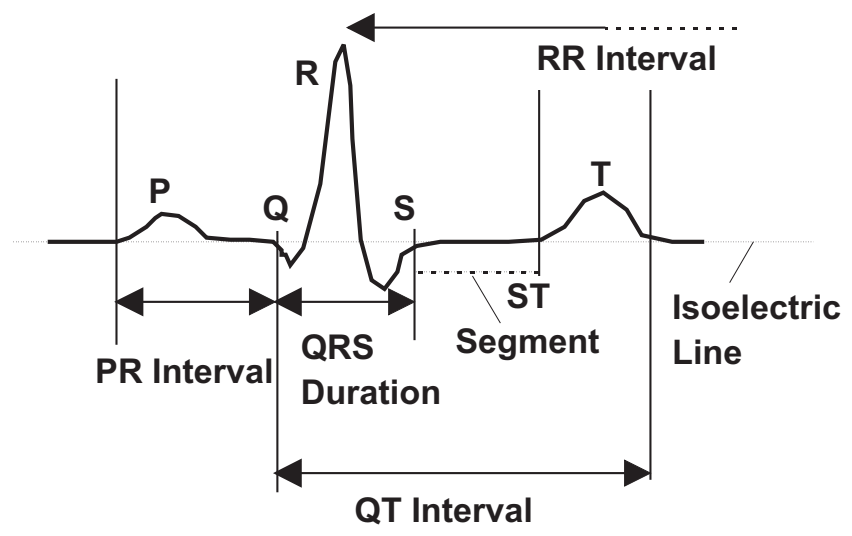

Fig. 1. Normal ECG delineation

Producing an algorithm for the detection of the $P$ wave, $Q R S$ complex and $T$ wave in an ECG is a difficult problem due to the time varying morphology of the signal subject to physiological conditions, moreover the localization of wave onsets and ends is much more difficult, as 
the signal amplitude is low at the wave boundaries and the noise level can be higher than the signal itself. A number of wavelet-based techniques have been proposed to detect these features. Senhadji et al (1995) compared the ability of three different wavelets transforms (Daubechies, spline and Morlet) to recognize and describe isolated cardiac beats (Senhadji \& Wendling, 2002). Sahambi et al. employed a first-order derivative of the Gaussian function as the wavelet for the characterization of ECG waveforms (Sahambi et al., 1997a;b). Moreover, wavelet-based QRS detection methods have been suggested by a variety of groups including Li et al (1995) who proposed a method based on finding the modulus maxima larger than a threshold obtained from the pre-processing of preselected initial beats, this threshold can be updated during the analysis to obtain a better performance (Li et al., 1995).

Kadambe et al. have described an algorithm which finds the local maxima of two consecutive dyadic wavelet scales, and compared them in order to classify local maxima produced by $R$ waves and by noise. Kadambe et al. report a sensitivity of $96.84 \%$ and a positive predictive value of $95.20 \%$ when tested on a limited data set (four 30 min tapes acquired from the American Heart Association (AHA) database) (Kadambe et al., 1999). Other work has been undertaken by Park et al (1998) using a wavelet adaptive filter to minimize the distortion of the ST-segment due to baseline wanderings. In a subsequent paper by Park et al (2001), a wavelet interpolation filter (WAF) is described for the removal of motion artefacts in the ST-segment of stress ECGs (Park et al., 2001). Furthermore, Martinez et al (2004) also utilize the algorithm of $\mathrm{Li}$ et al applying a dyadic wavelet transform to a robust ECG delineation system which identifies the peaks, onsets and offsets of the QRS complexes, and $P$ and $T$ waves. The QRS detector obtains a sensitivity and a positive predictivity of $99.8 \%$ in a very well-known MIT-BIH Arrhythmia Database (Martinez et al., 2004).

\section{Heart rate variability}

Rather than consider the local morphology of the whole ECG signal, many researchers have focused on the longer term temporal variability of the heartbeat, the analysis of which allows an assessment of autonomous nervous system activity. The analysis of heart rate variability (HRV) requires the sequence of timing intervals between beats, taken between each $R$ point on the $Q R S$ complex. This $R R$ interval can be plotted against time to give the $R R$ time series. In normal practice, however, ectopic beats are removed from the $R R$ series leaving only normal sinus beats: the $N N$ time series. It is this modified time series that is used in the analysis of HRV. The minute fluctuations present in the $N N$ intervals are used for assessing the influence of the autonomic nervous system components on the heart rate. Long range correlations and power law scaling have been found through the analysis of heartbeat dynamics. The heart rate and rhythm is largely under the control of the autonomic nervous system. Traditional spectral analysis of HRV has been reported to aid the understanding of the modulatory effects of neural mechanisms on the sinus node. There are three main spectral components in a traditional spectral calculation, they are generally classed as: very low frequency (VLF) ranging from 0.003 to $0.04 \mathrm{~Hz}$, low frequency (LF) ranging from 0.04 to $0.15 \mathrm{~Hz}$ and high frequency (HF) ranging from 0.15 to $0.4 \mathrm{~Hz}$ components. In addition, sometimes an ultra low frequency (ULF) is defined as spectral components with frequencies less than $0.003 \mathrm{~Hz}$. The relative contribution of vagal and sympathetic modulation of the heart rate is attributed to the distribution of spectral power in these bands. The most common of the techniques rely on the accurate determination of the temporal location of the $R$ wave based on signal matched filters or time-frequency decomposition methods. Over recent years, a number of groups have attempted to use wavelet-based methods to gain additional insight 
into the mechanisms controlling heart rate variability. The wavelet transform is partitioned into the HF, LF and VLF regions whereby temporal-spectral characteristics of the surface may then be investigated. Thurner et al have employed both Daubechies D10 and Haar wavelets in the analysis of human heartbeat intervals. They found that, at distinct wavelet scales, corresponding to the interval 16-32 heartbeats, the scale-dependent standard deviations of the wavelet coefficients could differentiate between normal patients and those with heart failure. Significantly, they report $100 \%$ accuracy for a standard 27 patient data set (Thurner et al., 1998). Further development of the technique is detailed in subsequent papers (Heniford et al., 1998; Seidensticker et al., 1998; Wiklund et al., 2011). Ivanov et al investigated the ECG signals acquired from subjects with sleep apnea, by sampling at an a scale equivalent to 8 heartbeats, they performed a local smoothing of the high-frequency variations in the signal in order to probe patterns of duration in the interval 30-60 s. The authors used the data to characterize the nonstationary heartbeat behaviour and elucidate phase interactions (Ivanov et al., 1996). Furthermore, this type of analysis has been applied to study myocardial ischemia, where a method for analysing HRV signals using wavelet transform was applied to obtain a time-scale representation for VLF, LF and HF bands using the orthogonal multiresolution pyramidal algorithm. Comparing a normality zone against the ischaemic episode, it was found a statistical significant increase in the LF and HF bands in the ischaemic episode, this index can be useful for the assessment of dynamic changes and patterns of HRV during myocardial ischaemia (Gamero et al., 2002).

\section{Cardiac arrhythmias}

A number of wavelet-based techniques have been proposed for the identification, classification and analysis of arrhythmic ECG signals. In 1997, Govindan described an algorithm for classifying bipolar electrograms from the right atrium of sheep into four groups: normal sinus rhythm, atrial flutter, paroxysmal atrial fibrillation and chronic atrial fibrillation. In this method, it was used a Daubechies D6 wavelet to preprocess the ECG data prior to classification using an artificial neural network. They found paroxysmal AF the most difficult to classify with a $77 \% \pm 9 \%$ average success rate and normal sinus rhythm the easiest, achieving $94 \% \pm 8 \%$ (Govindan et al., 1997). Using a raised cosine wavelet transform, Khadra et al undertoke a preliminary investigation of three arrhythmias: ventricular fibrillation (VF), ventricular tachycardia (VT) and atrial fibrillation (AF) Khadra et al. (1997), they developed an algorithm based on the scale-dependent energy content of the wavelet decomposition to classify the arrhythmias, distinguishing them from each other and normal sinus rhythm. Zhang et al proposed a novel arrhythmia detection method, based on a wavelet network, for use in implantable defibrillators, their system, originally developed as a model to identify relationships between concurrent epicardial cell action potentials and bipolar electrogram, detects the bifurcation point in the ECG where normal sinus rhythm degenerates into a pathological arrhythmia (ventricular fibrillation) (Zhang et al., 1999). Al-Fahoum and Howitt proposed a radial basis neural network for the automatic detection and classification of arrhythmias which employs preprocessing of the ECG using the Daubechies D4 wavelet transform, they reported $97.5 \%$ correct classification of arrhythmia from a dataset of 159 arrhythmia files from three different sources, with 100\% correct classification for both ventricular fibrillation and ventricular tachycardia (al Fahoum \& Howitt, 1999). Moreover, it has been already shown its potential for the detection of ventricular late potentials (Dickhaus et al., 1994; Khadrea et al., 1993; Meste et al., 1994). 
Morlet et al presented a Morlet wavelet-based method for the discrimination of patients prone to the onset of ventricular tachycardias (VTs), they found that the detection of strings of local maxima of the wavelet transform vector at or after $98 \mathrm{~ms}$ after the QRS onset point was a reasonable criterion for VT risk stratification in post-infarction patients. They reported achieving $85 \%$ specificity at $90 \%$ sensitivity for their patient group (Morlet et al., 1993). Englund et al studied the predictive value of wavelet decomposition of the signal averaged ECG in identifying patients with hypertrophic cardiomyopathy at increased risk of malignant ventricular arrhythmias or sudden death (Englund et al., 1998), wavelet analysis used in their study was undertaken subsequent to signal averaging of the beats. Thus intermittent local or transient aspects of the ECG can be lost to its interrogation. A later study by this group evaluated a number of wavelet decomposition parameters for their potential for risk stratification of patients with idiopathic dilated cardiomyopathy (Yi et al., 2000). They found that wavelet analysis was superior to time domain analysis for identifying patients at increased risk of clinical deterioration.

In addition, different wavelet analysis have been applied to Atrial fibrillation (AF). It is the most frequently found sustained cardiac arrhythmia in clinical practice. It is the most common cause of embolic stroke, and is associated with a doubling of overall mortality and morbidity from cardiovascular disease (Benjamin et al., 1998; Kannel et al., 1982). AF is characterized by an abnormal excitation of the atria, where the normal and regular atrial activation is substituted by several coexisting wavefronts that continuously depolarize the atrial cells (Allessie et al., 1995; Fuster et al., 2006). As a result, atrial activation is chaotic and disorganized, and consequently the atria are not able to be contracted in a regular rhythm. On the surface electrocardiogram (ECG), $P$ waves are no longer visible, being replaced by rapid oscillations or fibrillatory waves that vary in size, shape, and timing (Allessie et al., 1995; Bollmann et al., 1999). The ventricular response depends on the electrophysiological properties of the atrioventricular node, what results in an irregular and rapid ventricular rhythm. Fig. 2 represents an example of normal sinus rhythm and AF episodes.
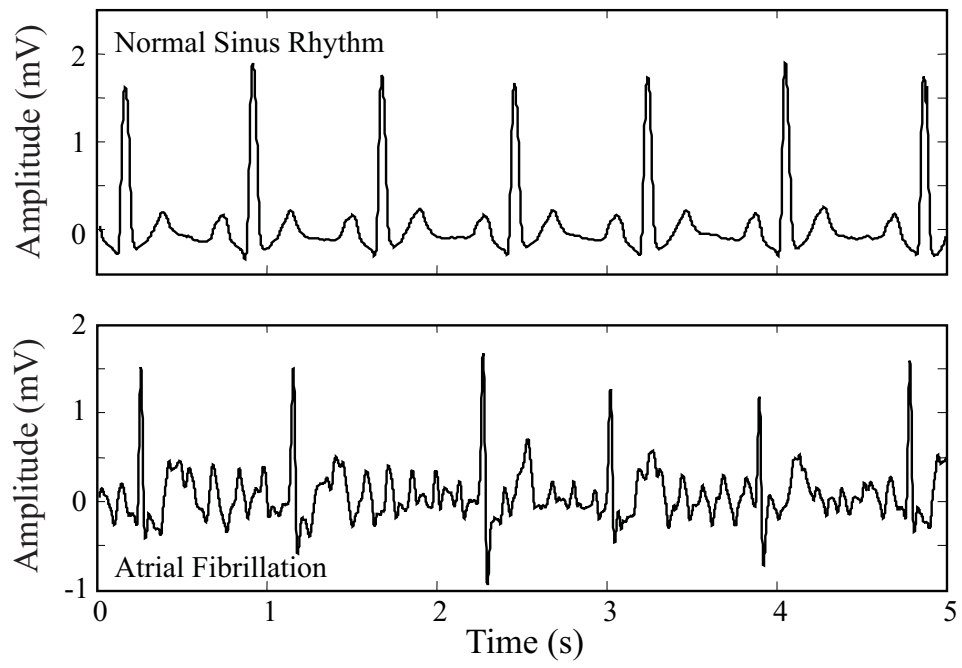

Fig. 2. Examples of normal sinus rhythm and AF episodes. 
Duverney et al have developed a combined wavelet transform-fractal analysis method for the automatic detection of AF from heart rate intervals (Roche et al., 2002). After training their method on healthy sinus rhythm and chronic AF ECGs, they achieved $96.1 \%$ sensitivity and $92.6 \%$ specificity for discriminating AF episodes in paroxysmal AF. A technique for the explanation of AF from within an ECG signal using a modulus maxima de-noising technique (Addison et al., 2000), where the modulus maxima lines, at this scale with a high proportion of the total energy within this scale are selected, are followed across scales and subtracted to leave a residual signal associated with both system noise and, more importantly, atrial activity. This time-frequency partitioning of the signal results in two components: one (1) containing combined low and high frequency components that correspond to large scale features in the signal, and a second (2) containing the remaining high frequency components that correspond to small scale AF features and noise. In practice, most applications are concerned with signal de-noising and hence the retention of component (1).

Furthermore, a study was conducted to analyze ECG signals from patients with persistent AF in order to extract reliable parameters to predict early AF recurrence after successful electrical cardioversion. The technique employed for ECG analysis was based on the wavelet transform, which have been successfully employed to solve other ECG problems. DWT analysis with biorthogonal family was applied, and the energy from different scales of detail coefficients of the descomposition was evaluated, the wavelet coefficients output at each subband may provide important information of the ECG signal, and they could be used in combination with appropriate statistical analysis tools in order to predict the risk of AF recurrence after successful electrical cardioversion. From this analysis, standard deviations of the coefficients in each subband were obtained, but its significance was lower than the cited parameter. The calculus of the ratio of the energy between different scales of the decomposition resulted statistically significant, however its capacity of prediction resulted lower than the continuous wavelet transform analysis, and the higher differences were obtained in the variable energy (eq. 6) in relation to some detail coefficients and the ratio between some scales of the decomposition (Cervigón et al., 2007). In addition, the effect of anaesthetic agents in restoration rhythm procedures during AF has not been investigated. It was evaluated the effects of a widely used anaesthetic agent (propofol) in the fibrillation patterns. Intra-atrial recordings belong patients diagnosed with AF were analyzed "before" (baseline) and "during" anaesthetic infusion. The goal of this study is to characterize the variation in atrial properties along the atria in both states. The wavelet variance of a time series on a scale by scale basis along the DWT decomposition, hence has considerable appeal when physical phenomena are analyzed in terms of variations operating over a range of different scales. As mother wavelet was used the haar wavelet and discrete wavelet transform partitioned the variance of a signal over 7 scales. The proposed methodology provide an additional approach to the understanding of the role of the anaesthetic, showing a decrease in the variance inter-scales during the anaesthetic infusion in the right atrium, with the opposite effect in the left atrium (i.e. a increase in the organization degree) (Cervigón et al., 2008).

\section{Conclusions}

Signal processing of the ECG has been already demonstrated its effectiveness to solve some clinical problems. In that sense, wavelet transform has emerged over recent years as a key time-frequency analysis and coding tool for the ECG. Indeed, its ability to localize simultaneously local spectral and temporal information within a signal. In addition, the fact that the wavelet transform exhibits different window sizes depending on the frequency 
band - broad at low frequencies and narrow at high frequencies - leads to an optimal time-frequency resolution in all frequency ranges. The coefficients output by the wavelet transform at each subband may provide important information of the ECG signal, and they could be used in combination with appropriate statistical analysis tools in order to predict different arrhythmias. It has been already shown its potential for feature extraction and discrimination between normal and abnormal cardiac patterns, detection of ventricular late potentials, characterization of beat-to-beat fluctuations in the heart rate under diverse physiological conditions, study of cardias arrhytmias, such as he risk of AF recurrence after successful electrical cardioversion etc.

In addition, its discrete form, the DWT provide the basis of powerful methodologies for partitioning pertinent signal components which serve as a basis for potent compression strategies.

The DWT has interesting mathematics and fits in with standard signal filtering and encoding methodologies. It produces few coefficients, where it is possible to recover the original signal, during the inverse transform process, without any loosing of energy. However, it exhibits non-stationarity and coarse time-frequency resolution.

DWT analysis of different signals has made possible the identification of pertinent features within the transform difficult, if not practically impossible. The non-stationarity of the DWT can also cause problems in terms of repeatability and robustness of the analysis, unless it particularly lends itself to an ensemble averaged method.

In conclusion, wavelet transform can be a helpful instrument to know more about the mechanisms of biological structure, it has been shown that inside biomedical signals, such as ECG signal contains hidden information that a tool such as wavelet transform could extract.

\section{References}

Thurner S., Markus F., \& Malvin T., (1998). Multiresolution Wavelet Analysis of Heartbeat Intervals Discriminates Healthy Patients from Those with Cardiac Pathology Physical Review Letters 7(80): 1544-9.

Abramovich, F., Grinshtein, V., Petsa, A. \& Sapatinas, T. (2009). On bayesian "testimation" and its application to wavelet thresholding.

Addison, P. S., Watson, J. N., Clegg, G. R., Holzer, M., Sterz, F. \& Robertson, C. E. (2000). Evaluating arrhythmias in ecg signals using wavelet transforms, IEEE Eng Med Biol Mag 19(5): 104-9.

al Fahoum, A. S. \& Howitt, I. (1999). Combined wavelet transformation and radial basis neural networks for classifying life-threatening cardiac arrhythmias, Med Biol Eng Comput 37(5): 566-73.

Allessie, M., Konings, K. \& Wijffels, M. (1995). Atrial arrhythmias: State of the art, DiMarco, J.P. and Prytowsky, E.N. Eds., Futura Publishing Company, Armonk, NY, chapter Electrophysiological mechanisms of atrial fibrillation, pp. 155-161.

Alshamali, A. \& Al-Fahoum, A. (2003). Comments on "an efficient coding algorithm for the compression of ecg signals using the wavelet transform", Biomedical Engineering, IEEE Transactions on 50(8): 1034-1037.

Benjamin, E., Wolf, P., RB, R. D., Silbershatz, H., Kannel, W. \& Levy, D. (1998). Impact of atrial fibrillation on the risk of death, Circulation (98): 946-952.

Bitzen, Alexander, Sternickel, Karsten, Lewalter, Thorsten, Schwab, Otto, J., Yang, Alexander, Schrickel, Wilko, J., Linhart, Markus, Wolpert, Christian, Jung, Werner, David, Peter, Luderitz, Berndt, Nickenig, Georg, Lickfett \& Lars (2007). Automatic p wave analysis 
over 24 hours in patients with paroxysmal or persistent atrial fibrillation, Annals of Noninvasive Electrocardiology 12(4): 306-315.

Bollmann, A., Sonne, K., Esperer., H., Toepffer, I., Langberg, J. \& Klein, H. (1999). Non-invasive assessment of fibrillatory activity in patients with paroxysmal and persistent atrial fibrillation using the holter ECG, Cardiovascular Research 44: 60-66.

Cervigón, R., Castells, F., Mateo, J., Moreno, J. \& Sánchez, C. (2008). Variance differences in atrial fibrillation during anaesthetic effect, Proc Conf IEEE Comput Cardiol.

Cervigón, R., Sánchez, C., Castells, F., Blas, J. \& Millet, J. (2007). Wavelet analysis of electrocardiograms to characterize recurrent atrial fibrillation, J Franklin Instit 344: 196-211.

Chen, J. \& Itoh, S. (1998). A wavelet transform-based ecg compression method guaranteeing desired signal quality, IEEE Trans Biomed Eng 45(12): 1414-9.

Crowe, J. A., Gibson, N. M., Woolfson, M. S. \& Somekh, M. G. (1992). Wavelet transform as a potential tool for ecg analysis and compression, J Biomed Eng 14(3): 268-72.

Daubechies, I. (1992). Ten Lectures on Wavelets (CBMS-NSF Regional Conference Series in Applied Mathematics), 1 edn, SIAM: Society for Industrial and Applied Mathematics.

Dickhaus, H., Khadrea, L. \& Brachmann, J. (1994). Time-frequency analysis of ventricular late potentials, Methods of Inform in Med 33(2): 187-195.

Donoho, D. \& Johnstone, J. (1994). Ideal spatial adaptation by wavelet shrinkage, Biometrika 81(3): 425-455.

Englund, A., Hnatkova, K., Kulakowski, P., Elliot, P. M., Malik, M. \& McKenna, W. J. (1998). Use of spectral turbulence analysis for the identification of patients at high risk for ventricular fibrillation and sudden death in patients with hypertrophic cardiomyopathy, Cardiology 90(2): 79-82.

Fuster, V., RydÈn, L. E., Cannom, D. S., Crijns, H. J., Curtis, A. B., Ellenbogen, K. A., Halperin, J. L., Heuzey, J.-Y. L., Kay, G. N., Lowe, J. E., Olsson, S. B., Prystowsky, E. N., Tamargo, J. L., Wann, S., Smith, S. C., Jacobs, A. K., Adams, C. D., Anderson, J. L., Antman, E. M., Halperin, J. L., Hunt, S. A., Nishimura, R., Ornato, J. P., Page, R. L., Riegel, B., Priori, S. G., Blanc, J.-J., Budaj, A., Camm, A. J., Dean, V., Deckers, J. W., Despres, C., Dickstein, K., Lekakis, J., McGregor, K., Metra, M., Morais, J., Osterspey, A., Tamargo, J. L., Zamorano, J. L., of Cardiology/American Heart Association Task Force on Practice Guidelines, A. C., of Cardiology Committee for Practice Guidelines, E. S., Association, E. H. R. \& Society, H. R. (2006). Acc/aha/esc 2006 guidelines for the management of patients with atrial fibrillation: a report of the american college of cardiology/american heart association task force on practice guidelines and the european society of cardiology committee for practice guidelines (writing committee to revise the 2001 guidelines for the management of patients with atrial fibrillation): developed in collaboration with the european heart rhythm association and the heart rhythm society., Circulation 114(7): e257-e354.

Gamero, L., Vila, J. \& Palacios, F. (2002). Wavelet transform analysis of heart rate variability during myocardial ischaemia, Medical and Biological Engineering and Computing 40: 72-78.

Geddes, L. A. \& Badylak, S. F. (1991). Power capability of skeletal muscle to pump blood, ASAIO Trans 37(1): 19-23.

Govindan, A., Deng, G. \& Power, J. (1997). Electrogram analysis during atrial fibrillation using wavelet and neural network techniques, Proc. SPIE 3169 pp. 557-562. 
Heniford, B. T., Senler, S. O., Olsofka, J. M., Carrillo, E. H. \& Bergamini, T. M. (1998). May-thurner syndrome: management by endovascular surgical techniques, Ann Vasc Surg 12(5): 482-6.

Ivanov, P. C., Rosenblum, M. G., Peng, C. K., Mietus, J., Havlin, S., Stanley, H. E. \& Goldberger, A. L. (1996). Scaling behaviour of heartbeat intervals obtained by wavelet-based time-series analysis, Nature 383(6598): 323-7.

Kadambe, S., Murray, R. \& Boudreaux-Bartels, G. F. (1999). Wavelet transform-based qrs complex detector, IEEE Trans Biomed Eng 46(7): 838-48.

Kaiser, G. (1994). A Friendly Guide to Wavelets, Birkhauser.

Kannel, W., Abbott, R. \& Savage, D. (1982). Epidemiological features of chronic atrial fibrillation and the risk of stroke. the framingham study, New England Journal of Medicine (306): 1018-1022.

Khadra, L., al Fahoum, A. S. \& al Nashash, H. (1997). Detection of life-threatening cardiac arrhythmias using the wavelet transformation, Med Biol Eng Comput 35(6): 626-32.

Khadrea, L., Dickhaus, H. \& Lipp, A. (1993). Representation of ecg-late potentials in the time frequency plane, J.Med Eng and Techcnol. 17(6): 228-231.

Kishimoto, K., Inoue, H., Hamada, M. \& Shibuya, T. (1995). Time frequency analysis of dispersive waves by means of wavelet transform, Journal of Applied Mechanics 62(4): 841-846.

Li, C., Zheng, C. \& Tai, C. (1995). Detection of ecg characteristic points using wavelet transforms, IEEE Trans Biomed Eng 42(1): 21-8.

Lio, P. (2003). Wavelets in bioinformatics and computational biology: state of art and perspectives, Bioinformatics 19(1): 2-9.

Mallat, S. (1989a). Multiresolution approximations and wavelet orthonormal bases of 12(r), Transactions of the American Mathematical Society 315(1).

Mallat, S. (1989b). A theory for multiresolution signal decomposition - the wavelet representation, IEEE Transactions on Pattern Analysis and Machine Intelligence 11: 674-693.

Mallat, S. (1998). A Wavelet Tour of Signal Processing, Academic Press.

Mallat, S. (1999). A Wavelet Tour of Signal Processing, Second Edition (Wavelet Analysis E Its Applications), 2 edn, Academic Press.

Martinez, J., Almeida, R, O. S., Rocha, A. \& Laguna, P. (2004). A wavelet-based ecg delineator: evaluation on standard databases, IEEE Transactions on Biomedical Engigneering 51: 570-581.

Meste, O., Rix, H., Caminal, P. \& Thakor, N. (1994). Ventricular late potentials characterization in time -frequency domain by means of a wavelet transform, IEEE Tras Biomed. Eng. 1: 625-634.

Miaou, S. G. \& Larn, J. H. (2000). Adaptive vector quantisation for electrocardiogram signal compression using overlapped and linearly shifted codevectors, Med Biol Eng Comput 38(5): 547-52.

Miaou, S.-G. \& Lin, C.-L. (2002). A quality-on-demand algorithm for wavelet-based compression of electrocardiogram signals, IEEE Trans Biomed Eng 49(3): 233-9.

Morlet, D., Peyrin, F., Desseigne, P., Touboul, P. \& Rubel, P. (1993). Wavelet analysis of high-resolution signal-averaged ecgs in postinfarction patients, J Electrocardiol 26(4): 311-20. 
Nikolaev, N., Gotchev, A., Egiazarian, K. \& Nikolov, Z. (2001). Suppression of electromyogram interference on the electrocardiogram by transform domain denoising, Med Biol Eng Comput 39(6): 649-55.

Park, K. L., Khil, M. J., Lee, B. C., Jeong, K. S., Lee, K. J. \& Yoon, H. R. (2001). Design of a wavelet interpolation filter for enhancement of the st-segment, Med Biol Eng Comput 39(3): 355-61.

Roche, F., Duverney, D., Court-Fortune, I., Pichot, V., Costes, F., Lacour, J.-R., Antoniadis, J. A., Gaspoz, J.-M. \& Barthelemy, J.-C. (2002). Cardiac interbeat interval increment for the identification of obstructive sleep apnea, Pacing Clin Electrophysiol 25(8): 1192-9.

Sahambi, J., Tandon, S. \& Bhatt, R. (1997a). Quantitative analysis of errors due to power-line interference and base-line drift in detection of onsets and offsets in ecg using wavelets, Medical and Biological Engineering and Computing 35(6): 747-751.

Sahambi, J., Tandon, S. \& Bhatt, R. (1997b). Using wavelet transforms for ecg characterization. an on-line digital signal processing system, IEEE Engineering in Medicine and Biology Magazine 16(1): 77-83.

Seidensticker, D., Wilcox, J. \& Gagne, P. (1998). Treatment of may-thurner syndrome with catheter-directed thrombolysis and stent placement, complicated by heparin-induced thrombocytopenia, Cardiovasc Surg 6(6): 607-13.

Senhadji, L. \& Wendling, F. (2002). Epileptic transient detection: wavelets and time-frequency approaches., Neurophysiologie clinique = Clinical neurophysiology 32(3): 175-192.

Strang, G. \& Nguyen, T. (1997). Wavelets and filter banks, Wellesley-Cambridge Press.

Thakor, N. V., Guo, X. R., Sun, Y. C. \& Hanley, D. F. (1993). Multiresolution wavelet analysis of evoked potentials, IEEE Trans Biomed Eng 40(11): 1085-94.

Tikkanen, P. (1999). Nonlinear wavelet and wavelet packet denoising of electrocardiogram signal., Biol Cybern 80(4): 259-267.

Wiklund, K., Fernández-Varea, J. M. \& Lind, B. K. (2011). A monte carlo program for the analysis of low-energy electron tracks in liquid water, Phys Med Biol 56(7): 1985-2003.

Yi, G., Hnatkova, K., Mahon, N. G., Keeling, P. J., Reardon, M., Camm, A. J. \& Malik, M. (2000). Predictive value of wavelet decomposition of the signal-averaged electrocardiogram in idiopathic dilated cardiomyopathy, Eur Heart J 21(12): 1015-22.

Zhang, X. S., Zhu, Y. S., Thakor, N. V., Wang, Z. M. \& Wang, Z. Z. (1999). Modeling the relationship between concurrent epicardial action potentials and bipolar electrograms, IEEE Trans Biomed Eng 46(4): 365-76. 


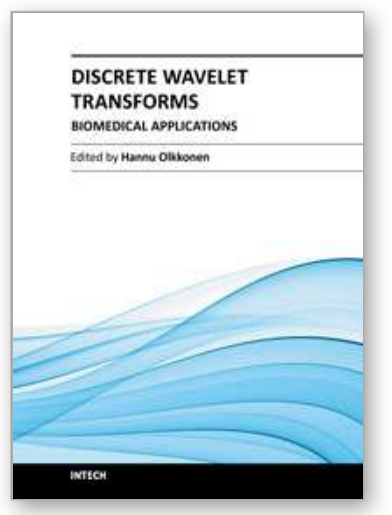

\author{
Discrete Wavelet Transforms - Biomedical Applications \\ Edited by Prof. Hannu Olkkonen
}

ISBN 978-953-307-654-6

Hard cover, 366 pages

Publisher InTech

Published online 12, September, 2011

Published in print edition September, 2011

The discrete wavelet transform (DWT) algorithms have a firm position in processing of signals in several areas of research and industry. As DWT provides both octave-scale frequency and spatial timing of the analyzed signal, it is constantly used to solve and treat more and more advanced problems. The present book: Discrete Wavelet Transforms - Biomedical Applications reviews the recent progress in discrete wavelet transform algorithms and applications. The book reviews the recent progress in DWT algorithms for biomedical applications. The book covers a wide range of architectures (e.g. lifting, shift invariance, multi-scale analysis) for constructing DWTs. The book chapters are organized into four major parts. Part I describes the progress in implementations of the DWT algorithms in biomedical signal analysis. Applications include compression and filtering of biomedical signals, DWT based selection of salient EEG frequency band, shift invariant DWTs for multiscale analysis and DWT assisted heart sound analysis. Part II addresses speech analysis, modeling and understanding of speech and speaker recognition. Part III focuses biosensor applications such as calibration of enzymatic sensors, multiscale analysis of wireless capsule endoscopy recordings, DWT assisted electronic nose analysis and optical fibre sensor analyses. Finally, Part IV describes DWT algorithms for tools in identification and diagnostics: identification based on hand geometry, identification of species groupings, object detection and tracking, DWT signatures and diagnostics for assessment of ICU agitation-sedation controllers and DWT based diagnostics of power transformers. The chapters of the present book consist of both tutorial and highly advanced material. Therefore, the book is intended to be a reference text for graduate students and researchers to obtain state-of-the-art knowledge on specific applications.

\title{
How to reference
}

In order to correctly reference this scholarly work, feel free to copy and paste the following:

Raquel Cervigón (2011). Biomedical Applications of the Discrete Wavelet Transform, Discrete Wavelet Transforms - Biomedical Applications, Prof. Hannu Olkkonen (Ed.), ISBN: 978-953-307-654-6, InTech, Available from: http://www.intechopen.com/books/discrete-wavelet-transforms-biomedicalapplications/biomedical-applications-of-the-discrete-wavelet-transform

\section{INTECH}

open science | open minds

\author{
InTech Europe \\ University Campus STeP Ri \\ Slavka Krautzeka 83/A
}

\section{InTech China}

Unit 405, Office Block, Hotel Equatorial Shanghai

No.65, Yan An Road (West), Shanghai, 200040, China 
51000 Rijeka, Croatia

Phone: +385 (51) 770447

Fax: +385 (51) 686166

www.intechopen.com
中国上海市延安西路65号上海国际贵都大饭店办公楼 405 单元 Phone: +86-21-62489820

Fax: +86-21-62489821 
(C) 2011 The Author(s). Licensee IntechOpen. This chapter is distributed under the terms of the Creative Commons Attribution-NonCommercialShareAlike-3.0 License, which permits use, distribution and reproduction for non-commercial purposes, provided the original is properly cited and derivative works building on this content are distributed under the same license. 